\title{
Research Data Storage Available to Researchers Throughout the U.S. via the TeraGrid
}

\author{
D. Scott McCaulay \\ Indiana University \\ 2711 East $10^{\text {th }}$ Street \\ Bloomington, IN \\ (812) 855-4716 \\ smccaula@indiana.edu
}

\author{
Matt R. Link \\ Indiana University \\ 2711 East $10^{\text {th }}$ Street \\ Bloomington, IN \\ (812) $855-6339$ \\ mrlink@indiana.edu
}

\begin{abstract}
Many faculty members at small to mid-size colleges and universities do important, high quality research that requires significant storage. In many cases, such storage requirements are difficult to meet with local resources; even when local resources suffice, data integrity is best ensured by maintenance of a remote copy. Via the nationally-funded TeraGrid, Indiana University offers researchers at colleges and universities throughout the US the opportunity to easily store up to $1 \mathrm{~TB}$ of data within the IU data storage system.

The TeraGrid is the National Science Foundation's flagship effort to create a national research cyberinfrastructure, and one key goal of the TeraGrid is to provide facilities that improve the productivity of the US research community generally. Providing facilities that improve the capacity and reliability of research data storage is an important part of this. This paper will describe the process for storing data at IU via the TeraGrid, and will in general discuss how this capability is part of a larger TeraGrid-wide data storage strategy.
\end{abstract}

\section{Categories and Subject Descriptors}

H.3.4 [Information Storage and Retrieval]: Systems and Software - distributed systems.

\section{General Terms}

Performance, Reliability, Security

Keywords

Storage: large-scale, Grid Computing, TeraGrid

\section{INTRODUCTION}

Access to sufficient and reliable data storage is a critical requirement of much academic research. It is well known that the NSF-funded TeraGrid provides researchers nationwide with tens

THIS SPACE RESERVED FOR COPYRIGHT PERMISSION BLOCK of millions of CPU hours of computational processing time annually. It is less well known, but perhaps more important to many researchers throughout the US, that the TeraGrid can be used as a vehicle to meet research data storage needs. The processes for requesting an allocation of storage space via the TeraGrid and for using the High Performance Storage System at Indiana University are both straightforward and described in this paper.

Utilizing storage resources provided through the TeraGrid provides a simple and cost-effective way of preventing unintentional loss of important research data by maintaining a copy at a secure, remote location. Indiana University is making available to TeraGrid users up to 1 Terabyte of storage (the equivalent of nearly 1,500 data CDs) on IU's HPSS-based Massive Data Storage System (MDSS). This paper presents the mechanisms by which this storage can be requested and utilized. By reading this paper, and following the steps described in it, you can obtain without cost to yourself the use of 1 TeraByte of secure, persistent storage via the TeraGrid, and use it to help meet your research storage needs. (For researchers who need more than $1 \mathrm{~TB}$ of storage, larger allocations are also possible as described in this paper; $1 \mathrm{~TB}$ requests are approved automatically).

\section{ALLOCATION \& AUTHENTICATION 2.1 TeraGrid Allocation Process}

Any researcher affiliated with an institution of higher education in the U.S. is eligible to request an allocation on the TeraGrid. An allocation provides you with accounts to access systems provided by the TeraGrid Resource Providers, and allots you a certain number of CPU hours to use on specific machines.

The TeraGrid allocation scheme has traditionally been geared toward the allocation of CPU hours for processing. The TeraGrid is planning to expand its allocation process to include requests for other scarce resources, including storage, visualization resources, and support. In the meantime, individual TeraGrid sites have developed their own policies for allocation of non-computational resources.

What this means in practice is that you can complete a very simple procedure to receive a small allocation of CPU time, and use that authorization to make use of storage systems provided for TeraGrid users by Indiana University. 
TeraGrid allocations come in three categories, based on the number of Service Units (SUs) requested. TeraGrid Service Units (SUs) represent one CPU hour on the original TeraGrid IA-64 cluster. Requests and awards are referred to by the name of the committee which reviews and approves them. Requests for the larger allocations, those greater than 30,000 (Medium Resource Allocation Committee or MRAC) and greater than 200,000 (Large Resource Allocation Committee or LRAC) are peer-reviewed with allocations determined and granted at quarterly and semi-annual meetings, respectively. The starter allocations are reviewed on an ongoing basis by the Development Allocation Committee (DAC), and any reasonable request by a qualified researcher will be approved.

Request for a TeraGrid Development Allocation (DAC) is done through the Partnerships Online Proposal System (POPS). POPS can be accessed at https://pops-submit.paci.org/ . A first-time user should use the "Create POPS login" screen, which requires very little information; basically just name, institution, phone, and email. Having created an ID, you can $\log$ in to the POPS submission system to request your allocation.

In order to obtain an allocation that will enable you to use the TeraGrid for storage, you should request a proposal type of "New", select the radio button for "TeraGrid only: $0-30,000$ Service Units" and continue. From the next screen, select the DAC-TG option. This opens a new proposal submission. The proposal consists of six screens, four of which are required. The proposal can be saved to be completed and submitted later.

The information required to complete the proposal includes personal contact information (name, address, email, phone), research information (abstract, field, keywords), resource request (CPU hours, number of processors), and a CV document. Optional additional information includes co-PI contact information and details of supporting grants.

If you are requesting a TeraGrid allocation to use only storage resources, you can request a minimal number of CPU hours and processors (1-8 processors, 1,000 SUs). TeraGrid DAC allocation requests are processed very quickly. Within a few weeks you should receive a packet of information instructing you how to access your new accounts. As part of your DAC allocation, you will receive accounts at all the TeraGrid sites.

\subsection{TeraGrid Authentication}

Because the TeraGrid is a distributed cyberinfrastructure that involves many different computing systems, you don't really "log on" to the TeraGrid; you "authenticate" yourself to the TeraGrid through the use of an X.509 certificate.

Once you receive your TeraGrid account information, you will still need to request an X.509 certificate. Several of the TeraGrid sites, including NCSA, SDSC, and TACC offer facilities to request certificates. You will only need to obtain one certificate for access to all TeraGrid sites. The "certificate" is composed of a set of files stored in your home directory on the system where you requested the certificate.

The exact process to request a certificate varies by site. For example, if you are logged in to an NCSA machine, you would type the command "ncsa-cert-request" at the command prompt and follow the instructions to enter your password and a new pass phrase to be used with the certificate. Each time you use the certificate, you will be asked to provide this pass phrase.

The TeraGrid user documentation provides a very good introduction and overview to the whole certificate process here: http://www.teragrid.org/userinfo/access/auth setup.php. This should provide sufficient information to get you through the process and, if not, email help@teragrid.org for additional assistance. Additional documentation on the X.509 certificate process is also available in the Indiana University Knowledge Base. To access this information, simply go to http://kb.iu.edu/ and enter "teragrid certificate" in the search box.

In order for your new X.509 certificate to be used for TeraGrid authentication, a Distinguished Name (DN) entry needs to be included in the grid-mapfile on each system where you wish to submit TeraGrid jobs. A DN is a text string that uniquely identifies you as a user and must be entered into a grid-mapfile, which is a list that associates DNs with local user names on a system. You only need to obtain an X.509 certificate once. However, the DN entry must be added to the grid-mapfile on each TeraGrid site that you wish to use. If your initial use of the TeraGrid will be limited to using Indiana University's facility then you will have to do this process just once - for Indiana University. The command to add your information to the system's grid-mapfile is "gx-map". Typing "gx-map -interactive" at the command prompt begins a session in which you can either type or paste your DN, or have the script find it in the .globus directory if it exists on the same machine. Documentation for the gx-map command is also available at the TeraGrid and IU Knowledge Base locations listed above.

Fortunately, the X.509 certification and adding the DN to the grid-mapfile is a one-time process, and once done they are done. From this point on, using the TeraGrid to store and retrieve files is quite straightforward. There is one more simple step for authentication which needs to be done for each TeraGrid session. You must simply type "grid-proxy-init" at the command line. This will prompt you for the pass phrase associated with your X.509 certificate. This is in some senses equivalent to the login process on computers you are already familiar with. When you type your pass phrase successfully, you will receive something called a proxy certificate that will allow you access to TeraGrid resources for a time period (by default) of 12 hours.

There is a certain amount of overhead involved in getting started with the TeraGrid; however, once you go through these steps, you will be ready to use IU's data storage facilities. And, after that, you may discover you can make good use of other Teragrid facilities to enhance your research capabilities, making the things you do now more efficient, and opening up new computing capabilities in support of your research activities.

\section{ACCESSING IU'S MDSS}

\subsection{What is the IU Massive Data Storage System?}

The storage that Indiana University provides to US researchers via the TeraGrid is based on tape storage in large-capacity tape systems under the control of a storage management software program called High Performance Storage System (HPSS). HPSS is hierarchical storage system software that manages access to 
very large amounts of data. HPSS was developed by IBM and five Department of Energy laboratories with contributions from other laboratories and universities worldwide. See http://www.hpsscollaboration.org/ for additional information. HPSS is used for extremely large data collections, some as large as 5 Petabytes.

People often think of access to data on tape as being slow. Because of the capabilities of the robotic tape silos in which data tapes are stored, and because of the high speed of data transfer to and from tapes in these systems, access to data is very rapid. Hardware upgrades currently underway on IU's MDSS system will result in even greater access speeds.

\subsection{Requesting an Account}

Your TeraGrid allocation will automatically create accounts for you on IU's computing resources, but access to the MDSS system must be requested specifically. Once you receive your account information, go to http://www.iu.teragrid.org/storage/ to request an account on our storage system.

IU has developed their own policy for providing storage to TeraGrid users. We are offering to make up to $1 \mathrm{~TB}$ available upon request, and to store the data for one year past the end of the user's TeraGrid allocation. You may also use the form to request larger allocations, which will be reviewed and acted upon (and we expect, generally approved) on an ongoing basis.

IU's implementation of HPSS as the Massive Data Storage System (MDSS) is unique in that it is the first and only HPSS system to implement distributed data movers. IU maintains geographically separate silos on its Bloomington and Indianapolis campuses. Users who store data in IU's HPSS system have the option of keeping two copies - one in Bloomington, one in Indianapolis - ensuring that the data are stored reliably even in the event of the destruction of one of the machine rooms.

\subsection{HSI Command Line Interface}

Access to HPSS can be done through a simple command line interface that provides basic commands to send and retrieve files or view a directory of files.

The software to provide the interface has been installed on systems at all the TeraGrid sites, so no matter where you log in, you can access your files stored at IU. The software is stored in the Community Software Area (CSA) of the TeraGrid. This provides a common space to install on all TeraGrid systems software that will be used by a community of users, but which is not a part of the Common TeraGrid Software Stack (CTSS), the software that is by default installed on all TeraGrid systems.

To begin an interactive session to store or retrieve files, type "\$TG_COMMUNITY/IUHPSS/bin/iuhsi" from the command prompt. Since you have already entered "grid-proxy-init", the authentication to the HPSS system will be done with no further entry required.

The "iuhsi" command will put you in the HSI environment, where you will be greeted by the ? prompt. Although many commands are supported, a lot can be accomplished just by using three of them: put, get and ls.

The command "put $<$ filename $>$ " will transfer the named file to the HPSS system.
The command "get <filename $>$ " will retrieve the named file from HPSS.

The "ls" command performs the same function as in Linux/Unix, listing the contents of the current HPSS directory. Many other Linux/Unix commands work exactly as expected in the HSI environment, including: $\mathrm{cp}$; $\mathrm{mv}$; $\mathrm{rm}$; $\mathrm{cd}$; and mkdir to name but a few.

Another set of commands perform directory commands on the local session rather than the HPSS side. The "lls" command does a local directory listing, "lcd" changes the local directory, and so on.

Other important commands are also intuitive. Typing "help" provides a list and descriptions of all available commands. Any of the commands "quit", "exit", or "end" will terminate the session.

\subsection{Future Plans and Continued Support}

The HSI command line interface is the only method available for accessing HPSS through the TeraGrid today. We are currently working on making additional interfaces available, including GridFTP and a Samba bridge that will let you access HPSS from your desktop as though it were a mountable file system.

IU is currently engaged in a major upgrade of their HPSS-based Massive Data Storage System, to be completed Fall 2006, which will result in a significant increase in capacity and even more importantly, tremendous improvement in access time.

Over time, the specifics of the commands and interfaces used to access the system will change. For updated instructions, visit the IU Knowledge Base at http://kb.iu.edu and search on "teragrid" and "storage". If the Knowledge Base does not answer your questions, you are invited to email rac@indiana.edu for further information.

\section{THE TERAGRID}

Indiana University is one of nine resource partners contributing to the TeraGrid, along with the National Center for Supercomputing Applications, Oak Ridge National Laboratory, Pittsburgh Supercomputing Center, Purdue University, San Diego Supercomputer Center, Texas Advanced Computing Center, Argonne National Laboratory, and the National Center for Atmospheric Research.

The TeraGrid was launched by the National Science Foundation in 2001. Indiana University was added as a resource partner in 2003. IU plans to continue through their participation in the TeraGrid what they have long done for local researchers - focus on data-centric science and support for the Life Sciences.

\section{SUMMARY}

This paper has described one piece of functionality that is being provided to the national research community via the TeraGrid. This functionality will be of interest and use to many scientists, artists, engineers, and clinical researchers throughout the U.S. The High Performance Storage System is a tape based system: it does one thing and it does that extremely well. It stores data on tape reliably, securely, and for long periods of time. Data can be written to tape or restored from tape easily using the HSI software. 
IU will provide $1 \mathrm{~TB}$ of data storage to any researcher or student doing a research project who applies for and receives any allocation of resources from the TeraGrid, including the DAC process outlined above. Our hope and expectation is that this service will indeed contribute toward meeting one of the goals set by the National Science Foundation for the TeraGrid - providing enhanced effectiveness and efficiencies for the broader research community of the United States and thus enhance research productivity

\section{ACKNOWLEDGMENTS}

IU's involvement in the TeraGrid, and the presentation of this material, is based upon work supported by the National Science Foundation under Grants No. 0833618, SCI451237, SCI535258, and SCI504075. IU received a significant grant-in-kind as part of its initial deployment of the massive data storage system. The deployment of IU's MDSS has also been supported by the Indiana Genomics Initiative and the Indiana METACyt Initiative, both supported through grants from the Lilly Endowment, Inc; by Shared University Research grants from IBM, Inc.; and by NSF grants 0116050 and 0521433. Any opinions, findings, and conclusions or recommendations expressed in this material are those of the authors and do not necessarily reflect the views of the National Science Foundation.

\section{REFERENCES}

[1] Stewart, C.A., Repasky, R., Hart, D., Papakhian, M., Shankar, A., Wernert,E., Arenson, A., and G. Bernbom. 2003. Advanced Information Technology Support For Life Sciences Research. Presented at the SIGUCCS 2003 Conference, Sept. 21-24, San Antonio, TX.

[2] Samuel, J.V., Peebles, C.S., Noguchi, T., Stewart, C.A. (2002). Gauging IT Support Strategies: User needs then and now. Paper presented at the SIGUCCS 2002 Conference, November 20-23, Providence, RI..

[3] NSF TeraGrid, http://teragrid.org/

[4] HPSS Collaboration, http://www.hpss-collaboration.org/ 